\title{
Scanning Surface Potential Microscopy of Light-Induced Electric Potential from Photosystem I and Photosystem I Reconstituted Proteoliposomes
}

\author{
I. Lee,* M. Rodriguez, T. Kuritz, E. T. Owens, and E. Greenbaum** \\ * Department of Electrical Engineering, University of Tennessee, Knoxville, Tennessee 37996-2100 \\ **Chemical Science Division, Oak Ridge National Laboratory, Oak Ridge, TN 37831-6194
}

\begin{abstract}
Although both processes of vision and photosynthesis are initiated by absorption of visible light, they are chemically and energetically different. In vision, light triggers a thermodynamically downhill reaction that is preloaded by dark metabolism. Photon absorption by rhodopsin activates a G-protein cascade leading to cyclic guanosine monophosphate (cGMP) hydrolysis, which in turn closes cation-specific channels to generate a nerve signal. In photosynthesis, on the other hand, absorption of photons by the special reaction center chlorophylls in Photosystems I and II (PSI, PSII) triggers charge separations across the photosynthetic membrane. This charge separation generates a voltage that is the source of Gibbs energy for the thermodynamically uphill reactions of green plant photosynthesis: oxidation of water to molecular oxygen and reduction of atmospheric carbon dioxide to sugars. We have presented a hybrid system, a new reaction that demonstrates photoactivation of mammalian cells with a plant photosynthetic light sensory system [1]. In this scheme, mammalian cells that possess no photoactivity are changed into photosensitive cells after the treatment with the PSI reaction centers, which were delivered by PSI proteoliposomes.
\end{abstract}

By using the scanning surface probe microscopy (SSPM) as a diagnostic tool, we report here the measurements of the surface potential of hydrogenated soy phosphatidylcholine/cholestrol proteoliposomes with reconstituted, functional photosystem I reaction centers. The PSIproteoliposomes were imaged with the combined techniques of tapping-mode atomic force microscopy (AFM) and SSPM, illustrated in Fig. 1 and Fig.2. The apparent range of liposome diameters was 70-100 nm. The AFM-SSPM technique uses a slender cantilever probe with a slightly blunt apex. It provides accurate voltage measurements but exaggerates lateral dimensions. The theory for this technique has been developed by Jacobs et al. [2]. Lee et al. [3-4] provide additional information on the techniques used for working with single PSI reaction centers. The oneto-one correspondence between the AFM and SSPM liposome images is evident in Fig. 1 anf Fig.2. The images were obtained under illumination with a diode laser at $670 \mathrm{~nm}$, near the absorption maximum of chlorophyll $(671 \mathrm{~nm})$ in PSI-proteoliposomes. The AFM image of Fig. 1 illustrates the gross geometric structure of the PSI-proteoliposome, whereas the electrostatic SSPM image [Fig. 2] reveals a finer grained pebble-like structure in the surface potential map, suggesting a close packing of the PSI reaction centers in the liposome membrane. The surface potential of the proteoliposomes was found to be in the range of 10-70 $\mathrm{mV}$ with particle-like structures on each liposome's surface which is $\sim 1 \mathrm{mV}$.

\section{References}

[1] T. Kuritz et al., IEEE Trans. on NanoBiosci. 4, No. 2 (2005) 196.

[2] H. O. Jacobs, et al., J. Appl. Phys. 84 (1998).

[3] I. Lee et al., J. Phys. Chem. B 104 (2000) 2439.

[4] I. Lee et al., J. Phys. Chem. B 107 (2003) 14225. 


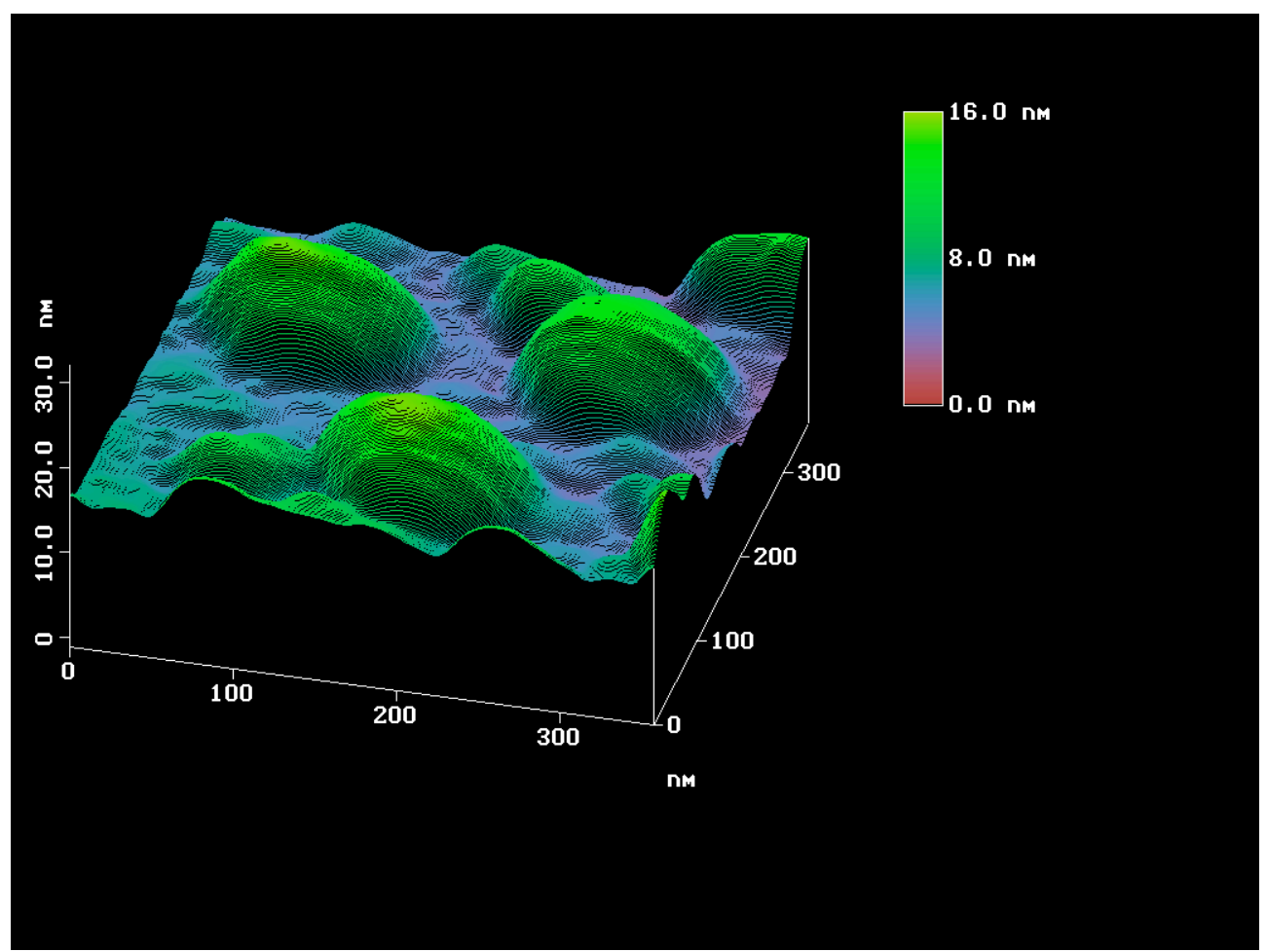

Fig. 1. PSI-proteoliposomes imaged by tapping-mode AFM.

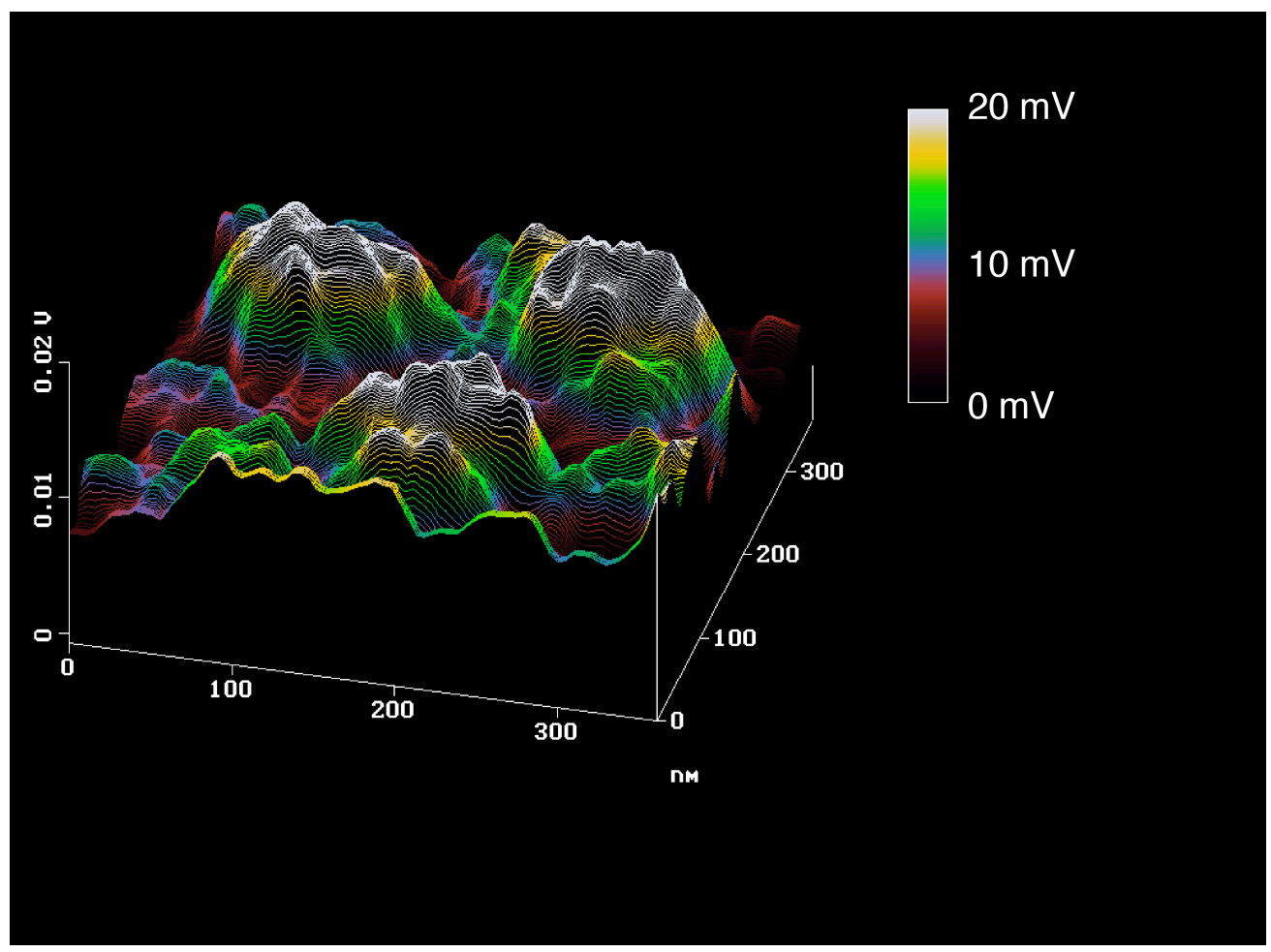

Fig. 2. PSI-proteoliposomes imaged by lift-mode SSPM. 